\title{
WIENER-HOPF FACTORIZATION FOR A FAMILY OF LÉVY PROCESSES RELATED TO THETA FUNCTIONS
}

\author{
A. KUZNETSOV, ${ }^{*}$ York University
}

\begin{abstract}
In this paper we study the Wiener-Hopf factorization for a class of Lévy processes with double-sided jumps, characterized by the fact that the density of the Lévy measure is given by an infinite series of exponential functions with positive coefficients. We express the Wiener-Hopf factors as infinite products over roots of a certain transcendental equation, and provide a series representation for the distribution of the supremum/infimum process evaluated at an independent exponential time. We also introduce five eight-parameter families of Lévy processes, defined by the fact that the density of the Lévy measure is a (fractional) derivative of the theta function, and we show that these processes can have a wide range of behavior of small jumps. These families of processes are of particular interest for applications, since the characteristic exponent has a simple expression, which allows efficient numerical computation of the Wiener-Hopf factors and distributions of various functionals of the process.
\end{abstract}

Keywords: Lévy process; Wiener-Hopf factorization; meromorphic function; infinite product; theta function

2010 Mathematics Subject Classification: Primary 60G51; 60E10

\section{Introduction}

Wiener-Hopf factorizaton and related fluctuation identities (see [4], [9], [12], and [18]), allow us to study various functionals of a Lévy process, such as extrema, first passage time, overshoots and undershoots, etc. There is a growing number of applications of these functionals and of the Wiener-Hopf factorization techniques in many areas of applied probability, most prominently in mathematical finance and insurance mathematics. However, the number of processes with two-sided jumps for which we can obtain explicit results on fluctuation identities is by no means large. In fact, until very recent times the only known examples consisted of a dense subclass of stable processes (see [8]), processes with phase-type distributed jumps (see [1] or [2]), and, more generally, processes having positive jumps with rational transform and arbitrary negative jumps (see [16]). In the last several years there have appeared a number of new results on the Wiener-Hopf factorization with varying degree of explicitness. First of all we would like to mention the class of Lamperti stable processes, which can be obtained by the Lamperti transformation (see [14]) from positive self-similar Markov processes, which are related to stable processes. The family of Lamperti stable processes is very interesting (and quite unique), since many fluctuation identities can be obtained in closed form in terms of elementary or special functions; see [5], [6], [13], and the references therein. Second, in [11]

Received 22 September 2009; revision received 24 July 2010.

* Postal address: Department of Mathematics and Statistics, York University, Toronto, ON, M3J 1P3, Canada.

Email address: kuznetsov@mathstat.yorku.ca

Research supported by the Natural Sciences and Engineering Research Council of Canada. 
we introduced a ten-parameter family of Lévy processes, for which the Wiener-Hopf factors can be identified in a semi-explicit form, more precisely as infinite products involving solutions to a certain transcendental equation. It is the goal of this paper to build on our earlier ideas and to extend the results in [11], as well as to provide new analytically tractable examples of Lévy processes with semi-explicit Wiener-Hopf factorization.

The main idea in [11] consisted of the following observation: if the characteristic exponent $\Psi(z)$ can be extended to a meromorphic function (which means that its only singularities in the complex plane are poles) and if we have some additional information about the asymptotic behavior of the solutions of the equation $\Psi(z)+q=0$, then we can obtain the Wiener-Hopf factors in essentially the same way as if $\Psi(z)$ was a rational function. Our first contribution in this paper is to show that the same technique works in a much more general setting: if the Lévy measure is an infinite series of exponential functions with positive coefficients then the Wiener-Hopf factorization and the distribution of extrema can be computed rather explicitly in terms of the solutions of the equation $\Psi(z)+q=0$. As we will see, the following property plays the most important role in the proof of our main result: the Lévy measure is an infinite series of exponential functions with positive coefficients if and only if, for every $q \geq 0$, the zeros and poles of $\Psi(z)+q$ lie on the imaginary axis and interlace. As our second contribution, we present several new families of Lévy processes which satisfy the above condition and which will be interesting for applications owing to their analytic tractability.

The paper is organized as follows: in Section 2 we prove Theorem 1 on the Wiener-Hopf factorization and distribution of extrema, while in Section 3 we construct five eight-parameter families of Lévy processes, for which the density of the Lévy measure is essentially the fractional derivative of the theta function. For each of these five families, we provide explicit formulae for the characteristic exponent and asymptotic expressions for the large roots of $q+\Psi(z)=0$, which are important for the efficient implementation of numerical algorithms.

\section{Main results}

First let us present several definitions and notation which will be used in this paper. We define the open/closed upper half-plane as

$$
\mathbb{C}^{+}=\{z \in \mathbb{C}: \operatorname{Im}(z)>0\}, \quad \overline{\mathbb{C}}^{+}=\{z \in \mathbb{C}: \operatorname{Im}(z) \geq 0\},
$$

and similarly for the negative half-plane and the positive/negative real half-line. We will study a one-dimensional Lévy process $X$ started from 0 , which is defined by the characteristic triple $(\mu, \sigma, \Pi)$. The characteristic exponent $\Psi(z)=-\ln \left(\mathrm{E}\left[\exp \left(\mathrm{i} z X_{1}\right)\right]\right)$ can be computed via the Lévy-Khintchine formula (see [4, Theorem 1.6, p. 4]) as follows:

$$
\Psi(z)=\frac{1}{2} \sigma^{2} z^{2}-\mathrm{i} \mu z-\int_{\mathbb{R}}\left(\mathrm{e}^{\mathrm{i} z x}-1-\mathrm{i} z x h(x)\right) \Pi(\mathrm{d} x), \quad z \in \mathbb{R},
$$

where $h(x)$ is the cutoff function (throughout in this paper, we will use $h(x) \equiv 0$ or $h(x) \equiv 1$, as the measure $\Pi(\mathrm{d} x)$ will have exponential tails). We define the supremum and infimum processes as

$$
S_{t}=\sup _{0 \leq s \leq t} X_{s}, \quad I_{t}=\inf _{0 \leq s \leq t} X_{s} .
$$

Wiener-Hopf factors (see [4], [9], [12], or [18]) are defined as

$$
\phi_{q}^{+}(z)=\mathrm{E}\left[\mathrm{e}^{\mathrm{i} z S_{e(q)}}\right] \quad \text { for } z \in \overline{\mathbb{C}}^{+}, \quad \phi_{q}^{-}(z)=\mathrm{E}\left[\mathrm{e}^{\mathrm{i} z I_{e(q)}}\right] \quad \text { for } z \in \overline{\mathbb{C}}^{-},
$$


where the random variable $e(q)$ is exponentially distributed with parameter $q>0$ and is independent of the process $X$.

In order to specify the Lévy measure $\Pi(\mathrm{d} x)$, we start with the four sequences of positive numbers $\left\{a_{n}, \rho_{n}, \hat{a}_{n}, \hat{\rho}_{n}\right\}_{n \geq 1}$, and assume that the sequences $\left\{\rho_{n}\right\}_{n \geq 1}$ and $\left\{\hat{\rho}_{n}\right\}_{n \geq 1}$ are strictly increasing, and that $\rho_{n} \rightarrow+\infty$ and $\hat{\rho}_{n} \rightarrow+\infty$ as $n \rightarrow+\infty$.

Assumption 1. The series $\sum_{n \geq 1} a_{n} \rho_{n}^{-2}$ and $\sum_{n \geq 1} \hat{a}_{n} \hat{\rho}_{n}^{-2}$ converge.

Next we define the function $\pi(x)$ as

$$
\pi(x)=\mathbf{1}(x>0) \sum_{n \geq 1} a_{n} \rho_{n} \mathrm{e}^{-\rho_{n} x}+\mathbf{1}(x<0) \sum_{n \geq 1} \hat{a}_{n} \hat{\rho}_{n} \mathrm{e}^{\hat{\rho}_{n} x} .
$$

It is easy to see that the above series converges for all $x \neq 0$, uniformly in $x$ on $\mathbb{R} \backslash(-\varepsilon, \varepsilon)$ for all $\varepsilon>0$. To check this, use Assumption 1 and the inequality $\exp \left(-\rho_{n} \varepsilon\right)<\rho_{n}^{-2}$, which holds for all $n$ large enough. It is clear that $\pi(x)$ is a positive function which decays exponentially as $|x| \rightarrow \infty$, and, as we show in the next proposition, Assumption 1 guarantees that $\pi(x)$ can be used to define a Lévy measure.

Proposition 1. Assumption 1 implies that $\int_{\mathbb{R}} x^{2} \pi(x) \mathrm{d} x<\infty$.

Proof. As mentioned above, for all $\varepsilon>0$, the first series in (2) converges uniformly for $x \in(\varepsilon, \infty)$; thus, we can integrate term by term and obtain

$$
\begin{aligned}
\int_{\varepsilon}^{\infty} x^{2} \pi(x) \mathrm{d} x & =\sum_{n \geq 1} a_{n} \rho_{n} \int_{\varepsilon}^{\infty} x^{2} \mathrm{e}^{-\rho_{n} x} \mathrm{~d} x \\
& =\sum_{n \geq 1} a_{n} \rho_{n}^{-2} \int_{\rho_{n} \varepsilon}^{\infty} u^{2} \mathrm{e}^{-u} \mathrm{~d} u \\
& <\sum_{n \geq 1} a_{n} \rho_{n}^{-2} \int_{\rho_{1} \varepsilon}^{\infty} u^{2} \mathrm{e}^{-u} \mathrm{~d} u \\
& <2 \sum_{n \geq 1} a_{n} \rho_{n}^{-2},
\end{aligned}
$$

where in the second step we have changed the variable of integration $x \mapsto u=\rho_{n} x$. We see that the integral on the left-hand side of the above inequality increases and is bounded as $\varepsilon \rightarrow 0^{+}$; therefore, it converges. The convergence of the integral over $(-\infty, 0)$ is proved similarly.

Proposition 1 allows us to use $\pi(x)$ as the density of a Lévy measure $\Pi(\mathrm{d} x)=\pi(x) \mathrm{d} x$. Note that, since $\pi(x)$ decays exponentially as $x \rightarrow \infty$, we can use the cutoff function $h(x) \equiv 1$ in (1); this will be the default choice throughout this section. The above property also allows us to work with the Laplace exponent, defined as

$$
\phi(z)=\ln \left(\mathrm{E}\left[\mathrm{e}^{z X_{1}}\right]\right)=-\Psi(-\mathrm{i} z) .
$$

Proposition 2. The Laplace exponent $\phi(z)$ is a real meromorphic function which has the following partial fraction decomposition:

$$
\phi(z)=\frac{1}{2} \sigma^{2} z^{2}+\mu z+z^{2} \sum_{n \geq 1} \frac{a_{n}}{\rho_{n}\left(\rho_{n}-z\right)}+z^{2} \sum_{n \geq 1} \frac{\hat{a}_{n}}{\hat{\rho}_{n}\left(\hat{\rho}_{n}+z\right)}, \quad z \in \mathbb{C} .
$$


Proof. Use (1), (2), and (3), integrate term by term, and rearrange the resulting infinite series.

Proposition 3. Assume that $q>0$. The equation $\phi(z)=q$ has solutions $\left\{\zeta_{n},-\hat{\zeta}_{n}\right\}_{n \geq 1}$, where $\left\{\zeta_{n}\right\}_{n \geq 1}$ and $\left\{\hat{\zeta}_{n}\right\}_{n \geq 1}$ are sequences of positive numbers which satisfy the following interlacing property:

$$
0<\zeta_{1}<\rho_{1}<\zeta_{2}<\rho_{2}<\cdots, \quad 0<\hat{\zeta}_{1}<\hat{\rho}_{1}<\hat{\zeta}_{2}<\hat{\rho}_{2}<\cdots .
$$

Proof. Using (4), we rewrite the equation $\phi(z)=q$ as

$$
z^{2} \sum_{n \geq 1} \frac{a_{n}}{\rho_{n}\left(\rho_{n}-z\right)}+z^{2} \sum_{n \geq 1} \frac{\hat{a}_{n}}{\hat{\rho}_{n}\left(\hat{\rho}_{n}+z\right)}=q-\frac{1}{2} \sigma^{2} z^{2}-\mu z .
$$

Denote the left-hand side of (6) as $\phi_{1}(z)$ and the right-hand side as $\phi_{2}(z)$. First let us check that there exists a solution to $(6)$ on intervals $\left(0, \rho_{1}\right)$ and $\left(-\hat{\rho}_{1}, 0\right)$. We observe that $\phi_{1}(0)=0$ and $\phi_{1}(z) \nearrow+\infty$ as $z \nearrow \rho_{1}$ or as $z \searrow-\hat{\rho}_{1}$; the function $\phi_{2}(z)$ is continuous and $\phi_{2}(0)>0$, and so all that is left to do is to apply the intermediate value theorem. Other intervals can be verified in a similar way.

It is important to emphasize that Proposition 3 does not claim that the sequences $\left\{\zeta_{n},-\hat{\zeta}_{n}\right\}_{n \geq 1}$ include all solutions to $\phi(z)=q$. The statement is that some solutions are real and that they interlace with the poles $\left\{\rho_{n},-\hat{\rho}_{n}\right\}_{n \geq 1}$. As we will see later, it is in fact true that there are no other solutions, but the proof of this statement is not trivial and requires some deep results from the theory of meromorphic functions.

Our first main result in this paper is the following theorem, which identifies the Wiener-Hopf factors and the distribution of extrema of the process $X$.

Theorem 1. Assume that $q>0$. Then, for $\operatorname{Re}(z)>0$,

$$
\phi_{q}^{+}(\mathrm{i} z)=\mathrm{E}\left[\mathrm{e}^{-z S_{e(q)}}\right]=\prod_{n \geq 1} \frac{1+z / \rho_{n}}{1+z / \zeta_{n}}, \quad \phi_{q}^{-}(-\mathrm{i} z)=\mathrm{E}\left[\mathrm{e}^{z I_{e(q)}}\right]=\prod_{n \geq 1} \frac{1+z / \hat{\rho}_{n}}{1+z / \hat{\zeta}_{n}} .
$$

The distribution of $S_{e(q)}$ can be identified as an infinite mixture of exponential distributions:

$$
\mathrm{P}\left(S_{e(q)}=0\right)=c_{0}, \quad \frac{\mathrm{d}}{\mathrm{d} x} \mathrm{P}\left(S_{e(q)} \leq x\right)=\sum_{n \geq 1} c_{n} \zeta_{n} \mathrm{e}^{-\zeta_{n} x}, \quad x>0,
$$

where the coefficients $\left\{c_{n}\right\}_{n \geq 0}$ are positive, satisfy $\sum_{n \geq 0} c_{n}=1$, and can be computed as

$$
c_{0}=\lim _{n \rightarrow+\infty} \prod_{k=1}^{n} \frac{\zeta_{k}}{\rho_{k}}, \quad c_{n}=\left(1-\frac{\zeta_{n}}{\rho_{n}}\right) \prod_{\substack{k \geq 1 \\ k \neq n}} \frac{1-\zeta_{n} / \rho_{k}}{1-\zeta_{n} / \zeta_{k}}
$$

The distribution of $-I_{e(q)}$ has the same form as above, with $\left\{\rho_{n}, \zeta_{n}\right\}$ replaced by $\left\{\hat{\rho}_{n}, \hat{\zeta}_{n}\right\}$.

Our proof of the Theorem 1 is based on two important results from the theory of meromorphic functions, which we present as theorems below. These two theorems combined together state that the Lévy measure has the form (2) if and only if, for every $q \geq 0$, the zeros and poles of the real meromorphic function $\phi(z)-q$ are real and interlace, and this function can be represented as an infinite product. 
Theorem 2. ([15, Theorem 1, p. 220].) A real meromorphic function $f(z)$ satisfies $f(z) \in \mathbb{C}^{+}$ for all $z \in \mathbb{C}^{+}$if and only if it can be represented in the form

$$
f(z)=c \frac{z-a_{0}}{z-b_{0}} \prod_{n \in \mathbb{Z} \backslash\{0\}} \frac{1-z / a_{n}}{1-z / b_{n}},
$$

where $c>0$, the zeros $a_{n}$ and the poles $b_{n}$ are real and satisfy the interlacing property

$$
b_{n}<a_{n}<b_{n+1}, \quad n \in \mathbb{Z},
$$

and $a_{-1}<0<b_{1}$.

Theorem 3. ([7, Theorem 1, p. 197].) A real meromorphic function $f(z)$ satisfies $f(z) \in \mathbb{C}^{+}$ for all $z \in \mathbb{C}^{+}$if and only if it can be represented in the form

$$
f(z)=\alpha z+\beta+\frac{B_{0}}{b_{0}-z}+\sum_{n \in \mathbb{Z} \backslash\{0\}} B_{n}\left(\frac{1}{b_{n}-z}-\frac{1}{b_{n}}\right),
$$

where the poles $b_{n}$ are real, $b_{n}<b_{n+1}$ and $b_{-1}<0<b_{1}, \alpha \geq 0, \beta \in \mathbb{R}, B_{n} \geq 0$ for $n \geq 0$, and the series $\sum B_{n} b_{n}^{-2}$ converges.

Proof of Theorem 1. First let us prove that the infinite products in (7) converge. The product $\prod b_{n}$ converges if and only if the series $\sum\left(b_{n}-1\right)$ converges; thus, we need to consider the series

$$
\sum_{n \geq 1}\left(\frac{1+z / \rho_{n}}{1+z / \zeta_{n}}-1\right)=z \sum_{n \geq 1} \frac{1}{1+z / \zeta_{n}}\left(\frac{1}{\rho_{n}}-\frac{1}{\zeta_{n}}\right) .
$$

Using the interlacing property (5) we find that

$$
0<\cdots<\rho_{2}^{-1}<\zeta_{2}^{-1}<\rho_{1}^{-1}<\zeta_{1}^{-1}
$$

thus, we obtain

$$
0<\sum_{n \geq 1}\left(\frac{1}{\zeta_{n}}-\frac{1}{\rho_{n}}\right)<\sum_{n \geq 1}\left(\frac{1}{\zeta_{n}}-\frac{1}{\zeta_{n+1}}\right)=\zeta_{1}^{-1}
$$

and conclude that the series on the right-hand side of (10) converges, which guarantees the convergence of the infinite products in (7).

Next, let us establish the following infinite product factorization:

$$
\frac{q}{q-\phi(z)}=\prod_{n \geq 1} \frac{1-z / \rho_{n}}{1-z / \zeta_{n}} \prod_{n \geq 1} \frac{1+z / \hat{\rho}_{n}}{1+z / \hat{\zeta}_{n}} .
$$

To prove this factorization, we use (4) and rewrite the function $(\phi(z)-q) / z$ in the form

$$
\frac{\phi(z)-q}{z}=\frac{1}{2} \sigma^{2} z+\mu-\frac{q}{z}+\sum_{n \geq 1} a_{n}\left(\frac{1}{\rho_{n}-z}-\frac{1}{\rho_{n}}\right)+\sum_{n \geq 1} \hat{a}_{n}\left(\frac{1}{-\hat{\rho}_{n}-z}-\frac{1}{-\hat{\rho}_{n}}\right) .
$$

Using the above equation and Theorem 3, we conclude that function $(\phi(z)-q) / z$ maps the upper half-plane into itself; therefore, applying Theorem 2 and Proposition 3 we find that

$$
\frac{\phi(z)-q}{z}=c \frac{z-\zeta_{1}}{z} \prod_{n \geq 1} \frac{1-z / \zeta_{n+1}}{1-z / \rho_{n}} \prod_{n \geq 1} \frac{1+z / \hat{\zeta}_{n}}{1+z / \hat{\rho}_{n}}
$$

which in turn implies (11). 
Let us introduce the functions

$$
f(z)=\prod_{n \geq 1} \frac{1+z / \rho_{n}}{1+z / \zeta_{n}}, \quad \hat{f}(z)=\prod_{n \geq 1} \frac{1+z / \hat{\rho}_{n}}{1+z / \hat{\zeta}_{n}}
$$

Using Theorem 2, we find that the real meromorphic function $z f(z)$ maps the upper halfplane into itself; thus, again, we apply Theorem 3 to find that $z f(z)$ has the partial fraction decomposition (9), which implies that $f(z)$ has the partial fraction decomposition

$$
f(z)=c_{0}+\sum_{n \geq 1} \frac{c_{n} \zeta_{n}}{\zeta_{n}+z}
$$

where $c_{n} \geq 0$ for $n \geq 0$ and the series $\sum c_{n}$ converges. Setting $z=0$ in the above equation we find that $\sum c_{n}=1$, and, therefore, $f(z)$ is the Laplace transform of the mixture of exponential distributions, which implies that $f(z)$ is the Laplace transform of a positive infinitely divisible random variable with zero drift. The same result holds for $\hat{f}(z)$. Equation (11) tells us that $q /(q-\phi(z))=f(-z) \hat{f}(z)$, and using the uniqueness of the Wiener-Hopf factorization (see [18, Theorem 45.2, p. 334]), we conclude that $\phi_{q}^{+}(\mathrm{i} z)=f(z)$ and $\phi_{q}^{-}(-\mathrm{i} z)=\hat{f}(z)$, thus obtaining (7).

In order to complete the proof, we need to establish only the formulae in (8). The expression for $c_{0}$ follows from the computation

$$
\mathrm{P}\left(S_{\mathrm{e}(q)}=0\right)=\lim _{z \rightarrow+\infty} \mathrm{E}\left[\mathrm{e}^{-z S_{\mathrm{e}(q)}}\right]=\lim _{z \rightarrow+\infty} \lim _{N \rightarrow+\infty} \prod_{n=1}^{N} \frac{1+z / \rho_{n}}{1+z / \zeta_{n}}=\lim _{N \rightarrow+\infty} \prod_{n=1}^{N} \frac{\zeta_{n}}{\rho_{n}},
$$

where in the last step we interchanged the two limits, which is possible owing to the fact that the limit

$$
\lim _{z \rightarrow+\infty} \prod_{n=1}^{N} \frac{1+z / \rho_{n}}{1+z / \zeta_{n}}
$$

converges uniformly in $N$. The expression for $c_{n}$ in (8) follows from (12) and the fact that $c_{n} \zeta_{n}$ is the residue of $f(z)$ at the pole $z=-\zeta_{n}$.

\section{Examples}

Theorem 1 gives us the Wiener-Hopf factors, and the distributions of $S_{e(q)}$ and $I_{e(q)}$ for any Lévy process whose Lévy measure is an infinite series of exponential functions with positive coefficients. All the formulae in Theorem 1 are based only on the zeros and the poles of the meromorphic function $\phi(z)-q$. The poles $\left\{\rho_{n},-\hat{\rho}_{n}\right\}_{n \geq 1}$ are usually known explicitly, but in order to find the zeros $\left\{\zeta_{n},-\hat{\zeta}_{n}\right\}_{n \geq 1}$, we have to solve the transcendental equation $\phi(z)=q$, and this has to be done numerically. It is clear that if we have to rely on the partial fraction decomposition (4) in order to evaluate $\phi(z)$, such numerical computations will be quite challenging, if not impossible. Thus, it is very important to find families of Lévy processes, for which the Lévy measure is an infinite series of exponentials, and at the same time the Laplace exponent can be computed in closed form. In [11] we introduced the $\beta$-family: a ten-parameter family of Lévy processes, for which the characteristic exponent can be computed in terms of the beta function. A very special subclass of the $\beta$-family is a five-parameter family of Lévy processes (see Section 3 of [11]), whose jump component behaves similarly to the 
normal inverse Gaussian process (see [3]), and the characteristic exponent is expressed in terms of elementary (trigonometric) functions. In this section we present five parametric families of Lévy processes, which have a number of desirable properties. The characteristic exponent is expressed in terms of rather simple functions, such as trigonometric functions or the digamma function $\psi(z)=\Gamma^{\prime}(z) / \Gamma(z)$ (see [10, Section 8.36, p. 902] for the definition and properties of the digamma function). The density of the Lévy measure decays exponentially at $\infty$ and has a singularity at 0 ,

$$
\pi(x) \sim a_{ \pm}|x|^{-\chi}, \quad x \rightarrow 0^{ \pm},
$$

of the order $\chi \in\left\{\frac{1}{2}, 1, \frac{3}{2}, 2, \frac{5}{2}\right\}$, thus 'covering' the complete range of admissible singularities $\chi \in(0,3)$. In particular, when $\chi=1$, we have a process of infinite activity of jumps but of finite variation, whose jump part is similar to the variance gamma process (see [17]), and when $\chi=2$, we obtain a process with an infinite variation of jumps, whose jump part is similar to the normal inverse Gaussian process.

To define these processes, we introduce the function $\Theta_{k}(x)$, defined as

$$
\Theta_{k}(x)=\delta_{k, 0}+2 \sum_{n \geq 1} n^{2 k} \mathrm{e}^{-n^{2} x}, \quad x>0,
$$

where $\delta_{k, 0}=1$ if $k=0$ and $\delta_{k, 0}=0$ otherwise. Note that $\Theta_{0}(x)=\theta_{3}\left(0, \mathrm{e}^{-x}\right)$ (see [10, Sections 8.18-8.19, p. 877] for the definition and properties of the theta functions $\left.\theta_{i}(z ; \tau)\right)$, and, for $k \in \mathbb{N}$, the function $\Theta_{k}(x)$ is just the $k$ th-order derivative of the theta function $\theta_{3}\left(0, \mathrm{e}^{-x}\right)$. In fact, results similar to those presented in this section can be established if we use $\theta_{2}\left(0, \mathrm{e}^{-x}\right)$ instead of $\theta_{3}\left(0, \mathrm{e}^{-x}\right)$.

Definition 1. For $0<\chi<3$ and $x \neq 0$, we define

$$
\pi_{\chi}(x)=\mathbf{1}(x>0) c_{1} \beta_{1} \mathrm{e}^{-\alpha_{1} x} \Theta_{k}\left(x \beta_{1}\right)+\mathbf{1}(x<0) c_{2} \beta_{2} \mathrm{e}^{\alpha_{2} x} \Theta_{k}\left(-x \beta_{2}\right),
$$

where $c_{i}, \alpha_{i}, \beta_{i}>0$ and $k=\chi-\frac{1}{2}$.

Proposition 4. The function $\pi_{\chi}(x)$ has the following asymptotics:

$$
\pi_{\chi}(x) \sim \begin{cases}\Gamma(\chi) c_{1} \beta_{1}^{1-\chi}|x|^{-\chi}, & x \rightarrow 0^{+} \\ \Gamma(\chi) c_{2} \beta_{2}^{1-\chi}|x|^{-\chi}, & x \rightarrow 0^{-}\end{cases}
$$

Proof. Let $h=\sqrt{x}$. Then, using the fact that a definite integral is the limit of the Riemann sum, we obtain, as $h \rightarrow 0^{+}$,

$$
\sum_{n \geq 1} n^{2 k} \mathrm{e}^{-n^{2} x}=h^{-1-2 k}\left[h \sum_{n \geq 1}(h n)^{2 k} \mathrm{e}^{-(h n)^{2}}\right]=x^{-1 / 2-k}\left[\int_{0}^{\infty} y^{2 k} \mathrm{e}^{-y^{2}} \mathrm{~d} y+o(1)\right] .
$$

To complete the proof, use Definition 1 and the above asymptotic relation.

Proposition 4 and Definition 1 guarantee that $\int_{\mathbb{R}} x^{2} \pi_{\chi}(x) \mathrm{d} x$ exists; thus, $\pi(x)$ can be used to define the density of a Lévy measure $\Pi_{\chi}(\mathrm{d} x)=\pi_{\chi}(x) \mathrm{d} x$. We define the Lévy process $X$ using the characteristic triplet $\left(\mu, \sigma, \Pi_{\chi}\right)$ and the Lévy-Khinchine formula (4).

Remark 1. Note that in a similar way we can construct Lévy processes with asymmetric behavior of small positive/negative jumps. We could extend Definition 1 to allow parameters $k_{1}$ and $k_{2}$ to control the order of the singularity of $\pi(x)$ as $x \rightarrow 0^{+}$and $x \rightarrow 0^{-}$, respectively. 
In the next five subsections we will present the results for the Lévy process $X$ with $\chi \in\left\{\frac{1}{2}, 1\right.$, $\left.\frac{3}{2}, 2, \frac{5}{2}\right\}$. The explicit formulae for the characteristic exponent $\Psi(z)$ are derived using the following approach. First we obtain the characteristic exponent $\Psi(z)$ for $\chi=\frac{1}{2}$ (this is just a simple application of formulae 6.162 of [10]) and $\chi=1$, in which case we have to use the following series expansion for the digamma function (see Equation 8.362.1 of [10]):

$$
\psi(x)=-\gamma-\frac{1}{x}+x \sum_{n \geq 1} \frac{1}{n(n+x)} .
$$

All the other cases, when $\chi \in\left\{\frac{3}{2}, 2, \frac{5}{2}\right\}$, can be easily derived from the above two by using Definition 1, the fact that $\Theta_{k+1}(x)=-\mathrm{d} \Theta_{k}(x) / \mathrm{d} x$ (which follows from (13)), and applying the following proposition (which can be easily established by integration by parts).

Proposition 5. (i) Assume that $\int_{\mathbb{R}^{+}} \mathrm{e}^{-\alpha x} \pi(x) \mathrm{d} x<\infty$. Then, for $z \in \mathbb{R}$,

$$
\int_{\mathbb{R}^{+}}\left(\mathrm{e}^{\mathrm{i} z x}-1\right) \mathrm{e}^{-\alpha x} \pi^{\prime}(x) \mathrm{d} x=(\alpha-\mathrm{i} z) f_{1}(z)-\alpha f_{1}(0),
$$

where $f_{1}(z)=\int_{\mathbb{R}^{+}} \mathrm{e}^{\mathrm{i} z x-\alpha x} \pi(x) \mathrm{d} x$.

(ii) Assume that $\int_{\mathbb{R}^{+}} x \mathrm{e}^{-\alpha x} \pi(x) \mathrm{d} x<\infty$. Then, for $z \in \mathbb{R}$,

$$
\int_{\mathbb{R}^{+}}\left(\mathrm{e}^{\mathrm{i} z x}-1-\mathrm{i} z x\right) \mathrm{e}^{-\alpha x} \pi^{\prime}(x) \mathrm{d} x=(\alpha-\mathrm{i} z) f_{2}(z)-\alpha z f_{2}^{\prime}(0),
$$

where $f_{2}(z)=\int_{\mathbb{R}^{+}}\left(\mathrm{e}^{\mathrm{i} z x}-1\right) \mathrm{e}^{-\alpha x} \pi(x) \mathrm{d} x$.

Asymptotic expansions for the large solutions of the equation $\phi(\zeta)=q$ are obtained using exactly the same technique as in the proof of the Theorem 5 of [11]; however, since the derivation of these expressions is rather lengthy and tedious, we have decided to omit it. The interested reader who decides to verify these formulae might want to use a symbolic computation software to make the algebraic manipulations more enjoyable.

All the formulae below involve the parameter $\sigma \geq 0$, positive numbers $\alpha_{i}, \beta_{i}$, and $c_{i}$ which define the Lévy measure $\Pi_{\chi}$ via Definition 1, and two additional parameters $\gamma$ and $\rho$. The parameter $\gamma$ is uniquely determined by the condition $\Psi(0)=0$ and the parameter $\rho$ is responsible for the linear drift. In all the cases when $\chi<2$, the jump part of the process has bounded variation; thus, we can take the cutoff function in the Lévy-Khintchine formula (1) as $h(x) \equiv 0$, and then we have $\rho=\mu$. When $\chi \geq 2$, we take $h(x) \equiv 1$ and then $\rho$ can be uniquely expressed in terms of the characteristic triplet $\left(\mu, \sigma, \Pi_{\chi}\right)$ via the condition $\mathrm{E}\left[X_{1}\right]=\mathrm{i} \Psi^{\prime}(0)=\mu$.

\subsection{The family of processes with $\chi=1 / 2$}

(i) The characteristic exponent can be computed as

$$
\begin{aligned}
\Psi(z)= & \frac{1}{2} \sigma^{2} z^{2}-\mathrm{i} \rho z-c_{1} \pi\left(\left(\alpha_{1}-\mathrm{i} z\right) \beta_{1}^{-1}\right)^{-1 / 2} \operatorname{coth}\left(\pi \sqrt{\left(\alpha_{1}-\mathrm{i} z\right) \beta_{1}^{-1}}\right) \\
& -c_{2} \pi\left(\left(\alpha_{2}+\mathrm{i} z\right) \beta_{2}^{-1}\right)^{-1 / 2} \operatorname{coth}\left(\pi \sqrt{\left(\alpha_{2}+\mathrm{i} z\right) \beta_{2}^{-1}}\right)+\gamma .
\end{aligned}
$$

(ii) For $n \geq 1$, we have $\rho_{n}=\alpha_{1}+\beta_{1}(n-1)^{2}$ and $\hat{\rho}_{n}=\alpha_{2}+\beta_{2}(n-1)^{2}$. 
(iii) If $\sigma \neq 0$ then the large positive solutions to $\phi(\zeta)=q$ satisfy

$$
\zeta \sim \beta_{2} n^{2}+\alpha_{2}+\frac{4}{\sigma^{2}} \frac{c_{2}}{\beta_{2}} n^{-4}+\frac{8}{\sigma^{4}} \frac{c_{2}}{\beta_{2}^{2}}\left(\mu-\alpha_{2} \sigma^{2}\right) n^{-6}+O\left(n^{-8}\right), \quad n \rightarrow+\infty .
$$

(iv) If $\sigma=0$ and $\mu \neq 0$, then the large positive solutions to $\phi(\zeta)=q$ satisfy

$$
\zeta \sim \beta_{2} n^{2}+\alpha_{2}-\frac{2 c_{2}}{\mu} n^{-2}+\frac{2}{\mu^{2}} \frac{c_{2}}{\beta_{2}}\left(\mu \alpha_{2}+\gamma+q\right) n^{-4}+O\left(n^{-5}\right), \quad n \rightarrow+\infty .
$$

\subsection{The family of processes with $\chi=1$}

(i) The characteristic exponent can be computed as

$$
\begin{aligned}
\Psi(z)= & \frac{1}{2} \sigma^{2} z^{2}-\mathrm{i} \rho z+c_{1} \psi\left(\mathrm{i} \sqrt{\left(\alpha_{1}-\mathrm{i} z\right) \beta_{1}^{-1}}\right)+c_{1} \psi\left(-\mathrm{i} \sqrt{\left(\alpha_{1}-\mathrm{i} z\right) \beta_{1}^{-1}}\right) \\
& +c_{2} \psi\left(\mathrm{i} \sqrt{\left(\alpha_{2}+\mathrm{i} z\right) \beta_{2}^{-1}}\right)+c_{2} \psi\left(-\mathrm{i} \sqrt{\left(\alpha_{2}+\mathrm{i} z\right) \beta_{2}^{-1}}\right)-\gamma .
\end{aligned}
$$

(ii) For $n \geq 1$, we have $\rho_{n}=\alpha_{1}+\beta_{1} n^{2}$ and $\hat{\rho}_{n}=\alpha_{2}+\beta_{2} n^{2}$.

(iii) If $\sigma \neq 0$ then the large positive solutions to $\phi(\zeta)=q$ satisfy

$$
\zeta \sim \beta_{2} n^{2}+\alpha_{2}+\frac{4}{\sigma^{2}} \frac{c_{2}}{\beta_{2}} n^{-3}+\frac{8}{\sigma^{4}} \frac{c_{2}}{\beta_{2}^{2}}\left(\mu-\alpha_{2} \sigma^{2}\right) n^{-5}+O\left(n^{-7}\right), \quad n \rightarrow+\infty .
$$

(iv) If $\sigma=0$ and $\mu \neq 0$, then the large positive solutions to $\phi(\zeta)=q$ satisfy

$$
\zeta \sim \beta_{2} n^{2}+\alpha_{2}-\frac{2 c_{2}}{\mu} n^{-1}+\frac{2}{\mu^{2}} \frac{c_{2}}{\beta_{2}}\left(2\left(c_{1}+c_{2}\right) \ln (n)+c_{0}\right) n^{-3}+O\left(n^{-4} \ln (n)\right)
$$

as $n \rightarrow+\infty$, where $c_{0}=\mu \alpha_{2}-\gamma+q+c_{1} \ln \left(\beta_{2} / \beta_{1}\right)$.

\subsection{The family of processes with $\chi=3 / 2$}

(i) The characteristic exponent can be computed as

$$
\begin{aligned}
\Psi(z)= & \frac{1}{2} \sigma^{2} z^{2}-\mathrm{i} \rho z+c_{1} \pi \sqrt{\left(\alpha_{1}-\mathrm{i} z\right) \beta_{1}^{-1}} \operatorname{coth}\left(\pi \sqrt{\left(\alpha_{1}-\mathrm{i} z\right) \beta_{1}^{-1}}\right) \\
& +c_{2} \pi \sqrt{\left(\alpha_{2}+\mathrm{i} z\right) \beta_{2}^{-1}} \operatorname{coth}\left(\pi \sqrt{\left(\alpha_{2}+\mathrm{i} z\right) \beta_{2}^{-1}}\right)-\gamma .
\end{aligned}
$$

(ii) For $n \geq 1$, we have $\rho_{n}=\alpha_{1}+\beta_{1} n^{2}$ and $\hat{\rho}_{n}=\alpha_{2}+\beta_{2} n^{2}$.

(iii) If $\sigma \neq 0$ then the large positive solutions to $\phi(\zeta)=q$ satisfy

$$
\zeta \sim \beta_{2} n^{2}+\alpha_{2}+\frac{4}{\sigma^{2}} \frac{c_{2}}{\beta_{2}} n^{-2}+\frac{8}{\sigma^{4}} \frac{c_{2}}{\beta_{2}^{2}}\left(\mu-\alpha_{2} \sigma^{2}\right) n^{-4}+O\left(n^{-6}\right), \quad n \rightarrow+\infty .
$$

(iv) If $\sigma=0$ and $\mu \neq 0$, then the large positive solutions to $\phi(\zeta)=q$ satisfy

$$
\zeta \sim \beta_{2} n^{2}+\alpha_{2}-\frac{2 c_{2}}{\mu}+\frac{2 \pi}{\mu^{2}} \frac{c_{1} c_{2}}{\sqrt{\beta_{1} \beta_{2}}} n^{-1}+O\left(n^{-2}\right), \quad n \rightarrow+\infty .
$$




\subsection{The family of processes with $\chi=2$}

(i) The characteristic exponent can be computed as

$$
\begin{aligned}
\Psi(z)= & \frac{1}{2} \sigma^{2} z^{2}-\mathrm{i} \rho z \\
& -c_{1}\left(\alpha_{1}-\mathrm{i} z\right) \beta_{1}^{-1}\left[\psi\left(\mathrm{i} \sqrt{\left(\alpha_{1}-\mathrm{i} z\right) \beta_{1}^{-1}}\right)+\psi\left(-\mathrm{i} \sqrt{\left(\alpha_{1}-\mathrm{i} z\right) \beta_{1}^{-1}}\right)\right] \\
& -c_{2}\left(\alpha_{2}+\mathrm{i} z\right) \beta_{2}^{-1}\left[\psi\left(\mathrm{i} \sqrt{\left(\alpha_{2}+\mathrm{i} z\right) \beta_{2}^{-1}}\right)+\psi\left(-\mathrm{i} \sqrt{\left(\alpha_{2}+\mathrm{i} z\right) \beta_{2}^{-1}}\right)\right]+\gamma .
\end{aligned}
$$

(ii) For $n \geq 1$, we have $\rho_{n}=\alpha_{1}+\beta_{1} n^{2}$ and $\hat{\rho}_{n}=\alpha_{2}+\beta_{2} n^{2}$.

(iii) If $\sigma \neq 0$ then the large positive solutions to $\phi(\zeta)=q$ satisfy

$$
\zeta \sim \beta_{2} n^{2}+\alpha_{2}+\frac{4}{\sigma^{2}} \frac{c_{2}}{\beta_{2}} n^{-1}+O\left(n^{-3} \ln (n)\right), \quad n \rightarrow+\infty .
$$

\subsection{The family of processes with $\chi=5 / 2$}

(i) The characteristic exponent can be computed as

$$
\begin{aligned}
\Psi(z)= & \frac{1}{2} \sigma^{2} z^{2}-\mathrm{i} \rho z-c_{1} \pi\left(\left(\alpha_{1}-\mathrm{i} z\right) \beta_{1}^{-1}\right)^{3 / 2} \operatorname{coth}\left(\pi \sqrt{\left(\alpha_{1}-\mathrm{i} z\right) \beta_{1}^{-1}}\right) \\
& -c_{2} \pi\left(\left(\alpha_{2}+\mathrm{i} z\right) \beta_{2}^{-1}\right)^{3 / 2} \operatorname{coth}\left(\pi \sqrt{\left(\alpha_{2}+\mathrm{i} z\right) \beta_{2}^{-1}}\right)+\gamma .
\end{aligned}
$$

(ii) For $n \geq 1$, we have $\rho_{n}=\alpha_{1}+\beta_{1} n^{2}$ and $\hat{\rho}_{n}=\alpha_{2}+\beta_{2} n^{2}$.

(iii) If $\sigma \neq 0$ then the large positive solutions to $\phi(\zeta)=q$ satisfy

$$
\zeta \sim \beta_{2} n^{2}+\alpha_{2}+\frac{4}{\sigma^{2}} \frac{c_{2}}{\beta_{2}}-\frac{8 \pi}{\sigma^{4}} \frac{c_{1} c_{2}}{\left(\beta_{1} \beta_{2}\right)^{3 / 2}} n^{-1}+O\left(n^{-2}\right), \quad n \rightarrow+\infty .
$$

(iv) If $\sigma=0$ then the large positive solutions to $\phi(\zeta)=q$ satisfy

$$
\zeta \sim \beta_{2}\left(n+w_{0}\right)^{2}+\alpha_{2}+\frac{2 \rho}{\pi^{2}} \frac{c_{2} \beta_{2}^{2} \beta_{1}^{3}}{c_{1}^{2} \beta_{2}^{3}+c_{2}^{2} \beta_{1}^{3}}+O\left(n^{-1}\right), \quad n \rightarrow+\infty,
$$

where

$$
w_{0}=\frac{1}{\pi} \arctan \left(\frac{c_{2} \beta_{1}^{3 / 2}}{c_{1} \beta_{2}^{3 / 2}}\right)
$$

\section{Conclusion}

In this paper we have extended the results of [11] in several directions. First of all, for a very large class of Lévy processes (having infinitely many parameters), we have proved that the Wiener-Hopf factors can be expressed as infinite products of linear factors and that the distribution of $S_{e(q)}$ and $I_{e(q)}$ can be identified as an infinite mixture of exponential distributions. Second, we have introduced five eight-parameter families of Lévy processes, which have a wide range of behavior of small jumps, including one family having jumps of finite activity (the density of the Lévy measure has a singularity at 0 of order $\chi=\frac{1}{2}$ ), two families with jumps of infinite activity but finite variation $\left(\chi=1\right.$ or $\left.\chi=\frac{3}{2}\right)$, and two families with jumps of infinite variation $\left(\chi=2\right.$ or $\left.\chi=\frac{5}{2}\right)$. We have also derived precise asymptotic expressions for the large 
solutions to $\phi(z)=q$, which are very useful for numerical computations. The characteristic exponents for these five families of processes have rather simple forms, especially when $\chi$ is a half-integer, in which case $\Psi(z)$ is given in terms of elementary trigonometric functions. This fact and the availability of efficient numerical schemes for computing the Wiener-Hopf factors and the distribution of extrema make these processes very interesting for mathematical modeling, in particular in the areas of mathematical finance and insurance mathematics.

\section{Acknowledgements}

I am grateful to James Langley for helpful suggestions related to the proof of Theorem 1. I would also like to thank Andreas Kyprianou and Juan Carlos Pardo for many stimulating and insightful discussions.

\section{References}

[1] Asmussen, S. (2000). Ruin Probabilities. World Scientific, River Edge, NJ.

[2] Asmussen, S., Avram, F. and Pistorius, M. R. (2004). Russian and American put options under exponential phase-type Lévy models. Stoch. Process. Appl. 109, 79-111.

[3] Barndorff-Nielsen, O. E. (1997). Normal inverse Gaussian distribution and stochastic volatility modelling. Scand. J. Statist. 24, 1-13.

[4] Bertoin, J. (1996). Lévy Processes. Cambridge University Press.

[5] Caballero, M. E. and Chaumont, L. (2006). Conditioned stable Lévy processes and the Lamperti representation. J. Appl. Prob. 43, 967-983.

[6] Chaumont, L., Kyprianou, A. E. And Pardo, J. C. (2009). Some explicit identities associated with positive self-similar Markov processes. Stoch. Process. Appl. 119, 980-1000.

[7] Čhebotarev, N. G. And MeǏman, N. N. (1949). The Routh-Hurwitz problem for polynomials and entire functions. Real quasipolynomials with $r=3, s=1$. Trudy Mat. Inst. Steklov 26, 3-331 (in Russian).

[8] Doney, R. A. (1987). On Wiener-Hopf factorization and the distribution of extrema for certain stable processes. Ann. Prob. 15, 1352-1362.

[9] Doney, R. A. (2007). Fluctuation Theory for Lévy Processes (Lecture Notes Math. 1897). Springer, Berlin.

[10] Gradshteyn, I. S. And Ryzhik, I. M. (2007). Table of Integrals, Series and Products, 7th edn. Academic Press, San Diego, CA.

[11] Kuznetsov, A. (2010). Wiener-Hopf factorization and distribution of extrema for a family of Lévy processes. Ann. Appl. Prob. 20, 1801-1830.

[12] Kyprianou, A. E. (2006). Introductory Lectures on Fluctuations of Lévy Processes with Applications. Springer, Berlin.

[13] Kyprianou, A. E., Pardo, J. C. And Rivero, V. (2010). Exact and asymptotic $n$-tuple laws at first and last passage. Ann. Appl. Prob. 20, 522-564.

[14] Lamperti, J. (1972). Semi-stable Markov processes. I. Z. Wahrscheinlichkeitsth 22, 205-225.

[15] Levin, B. YA. (1996). Lectures on Entire Functions (Trans. Math. Monogr. 150). American Mathematical Society, Providence, RI.

[16] Lewis, A. L. AND Mordecki, E. (2008). Wiener-Hopf factorization for Lévy processes having positive jumps with rational transforms. J. Appl. Prob. 45, 118-134.

[17] Madan, D. B. And Seneta, E. (1990). The Variance Gamma (VG) model for share market returns. J. Business 63, 511-524.

[18] Sato, K.-I. (1999). Lévy Processes and Infinitely Divisible Distributions (Camb. Stud. Adv. Math. 68). Cambridge University Press. 